\title{
SENSOR AND DATA FUSION CONTEST: TEST IMAGERY TO COMPARE AND COMBINE AIRBORNE SAR AND OPTICAL SENSORS FOR MAPPING
}

\author{
Olaf HELLWICH, Andreas REIGBER, Hartmut LEHMANN \\ Technical University Berlin, D-10623 Berlin, Germany \\ Department for Photogrammetry and Cartography \\ Phone: +49-30-31422796, Fax: +49-30-31421104 \\ E-mail: hellwich@fpk.tu-berlin.de \\ Working Group III/6
}

KEY WORDS: Data fusion, Multi-spectral data, Radar, SAR, Multi-temporal, Object recognition

\begin{abstract}
Presently, a data fusion contest is conducted to compare the potential of airborne SAR with optical sensors for mapping applications. The goal of the test is to answer two questions: (1) Can state-of-the-art airborne SAR compete with optical sensors in the mapping domain? (2) What can be gained when SAR and optical images are used in combination, i.e., when methods for information fusion are applied? The test is organized in the framework of the IEEE GRSS data fusion technical committee (DFC), ISPRS working group III/6 "Multi-Source Vision”, which both have strong relations with scientists, active in research on sensor fusion and automation in mapping, and - as the provider of the main organizational framework - the European Organization for Experimental Photogrammetric Research (OEEPE), which is the European research platform of national mapping agencies and other institutions, regarding technology developments to optimize the use of core data in a geoinformation infrastructure context. In the preparatory phase of the test, which has been started on the occasion of IGARSS 2001, test data has been collected and the scope of object extraction for mapping has been defined. The outcome of this phase, i.e. the test imagery to be used in the contest and its potential for mapping is presented in this paper.
\end{abstract}

\section{DATA}

A data fusion contest, titled "Information for Mapping from Airborne SAR and Optical Imagery", is organized under the umbrella of OEEPE, IEEE GRSS DFC, and ISPRS WG III/6 and will be started in summer 2002. The contest has been announced on the occasion of the International Geoscience and Remote Sensing Symposium 2001 in Sydney (1). In the mean time, existing test imagery from three test sites has been collected and the object types to be extracted from the imagery have been defined based on an intensive discussion with parties interested in the investigation.

In order to be able to map objects, relevant for map scales of about 1:25,000, with sufficient correctness, completeness, accuracy, and robustness, it is advisable to use optical imagery with a resolution of $1 \mathrm{~m}$, when automatic image analysis methods are to be applied. The reason for the use of such a high resolution ( $1 \mathrm{~m}$ in a scale of $1: 25,000$ corresponds to only $0.04 \mathrm{~mm}$ ) is that it is difficult to interprete airborne imagery, when objects in complex environments are to be extracted. Another reason is that topographic maps often contain linear objects, which have a width of only 3 to $5 \mathrm{~m}$.

When SAR imagery is to be used instead of optical imagery, speckle should be eliminated at least partially. Therefore, the SAR imagery should have a nominally higher resolution than the optical imagery. This is why it is planned to use for the mapping contest only SAR imagery of the worlds highest resolving airborne SAR systems presently publically available.
In order to also account for the large variety of modern SAR systems, different frequencies as well as polarimetric data were selected. Certainly, there are more parameters strongly influencing the appearance of topographic objects in SAR imagery - such as incidence angle and direction. In spite of their importance regarding object appearance, these parameters could not be considered for the selection of appropriate data, as the high demands of the contest, regarding resolution and other more practical matters, such as data costs and copyright, are already quite restricting. Nevertheless, the authors are convinced that the image data will provide a very good opportunity to investigate the applicability of SAR for mapping purposes.

The test areas to be investigated were selected neither to be of a too complex nor to be of a too simple structure. For instance, very densely built-up urban regions can hardly be found in the test data. They certainly would have posed a hard challenge for SAR imaging, so that it seems appropriate not to investigate them intensively at this stage of the test. Therefore, the test areas selected mainly include agricultural and forested rural areas as well as industrial areas with large flat buildings. They are located in Central Europe and Scandinavia.

The objects to be extracted from the imagery are roads, built-up areas, forests, agricultural fields, lakes, rivers, and railroads. Forests and agricultural fields should be subdivided into different types. The results of object extraction will be evaluated on the basis of different national mapping standards. 


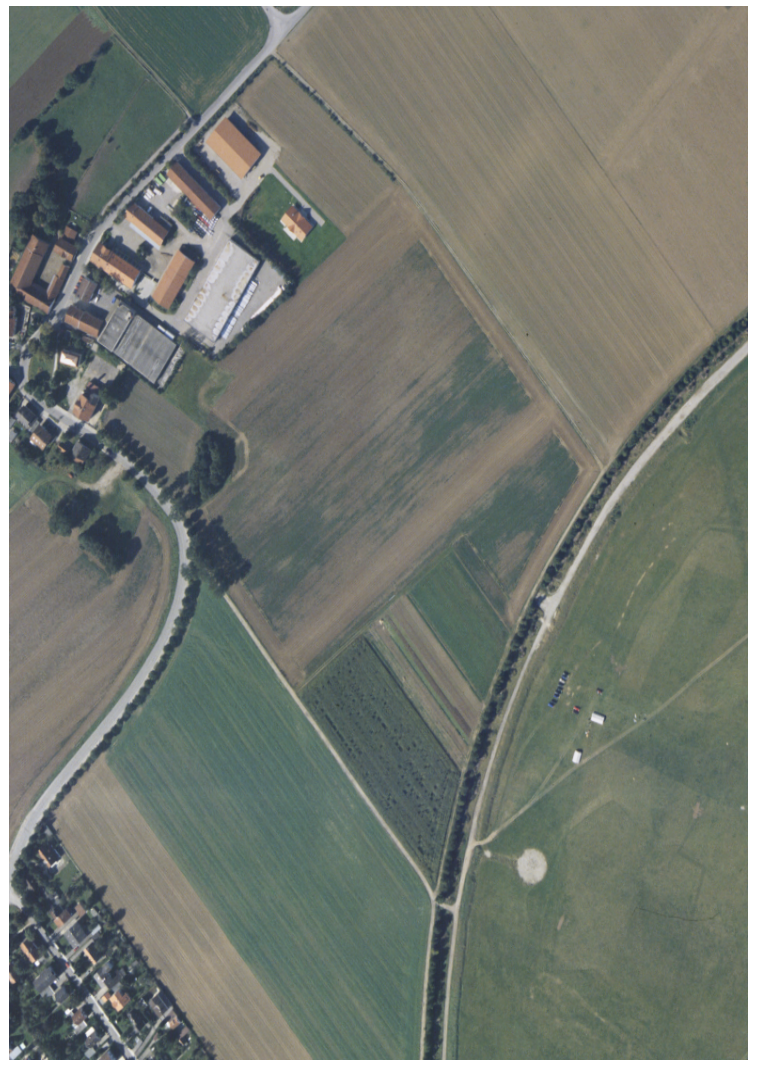

a)

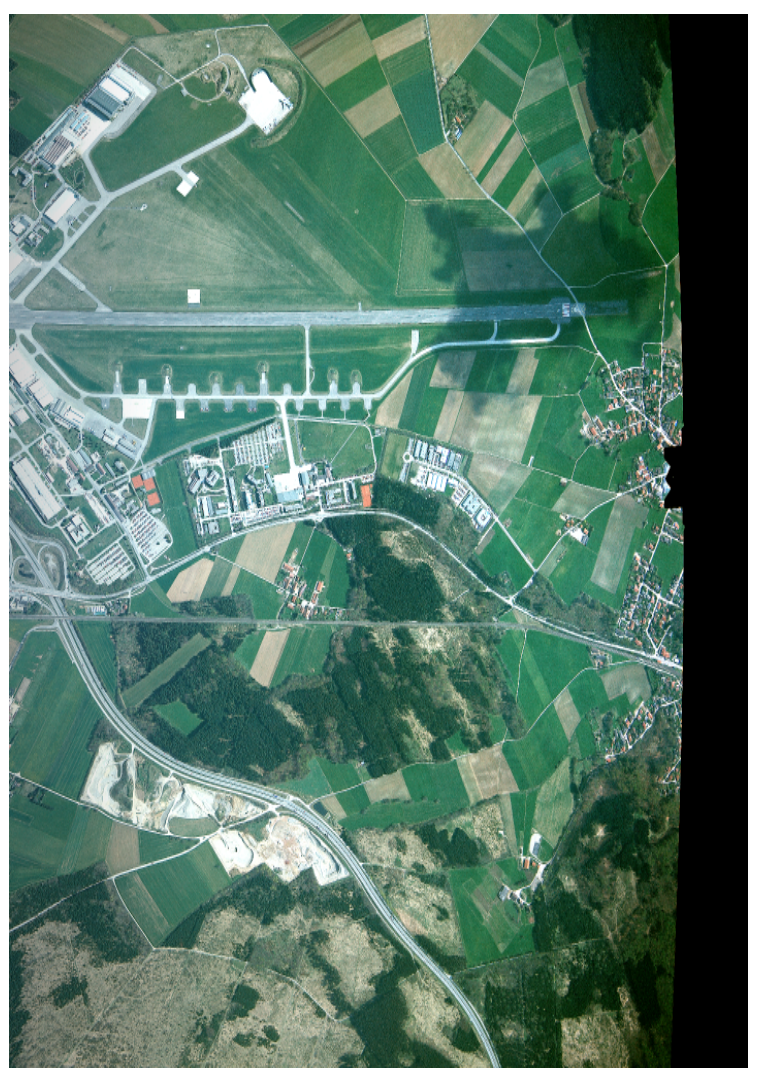

c)

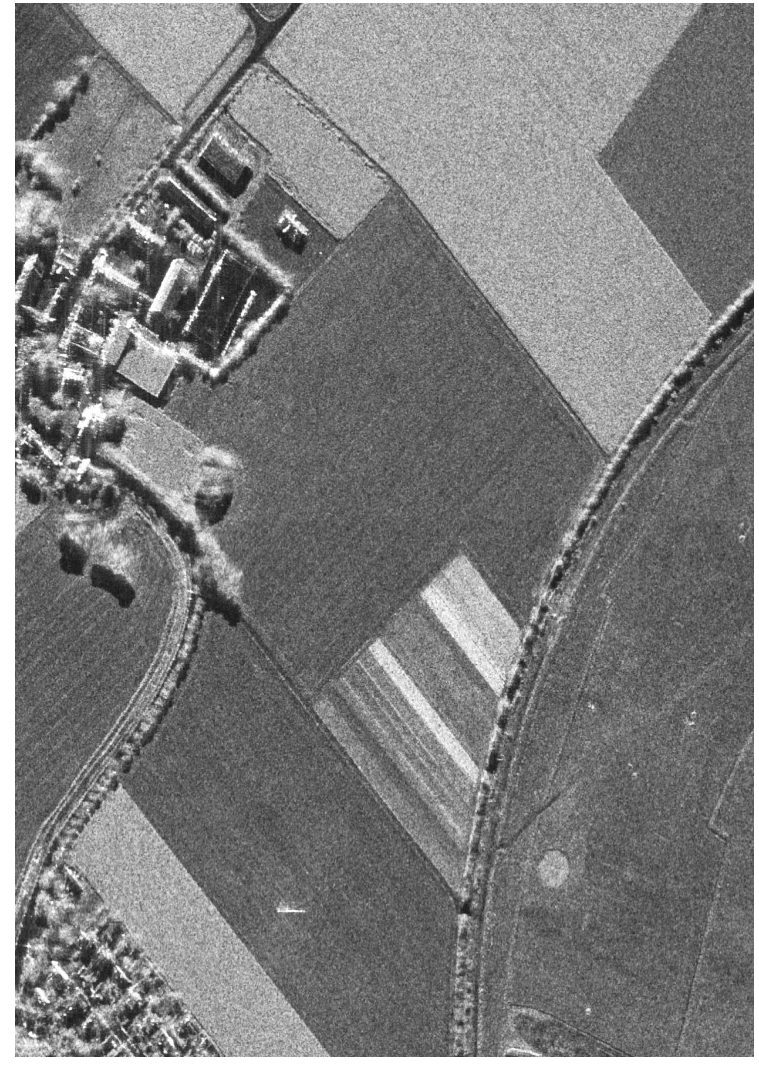

b)

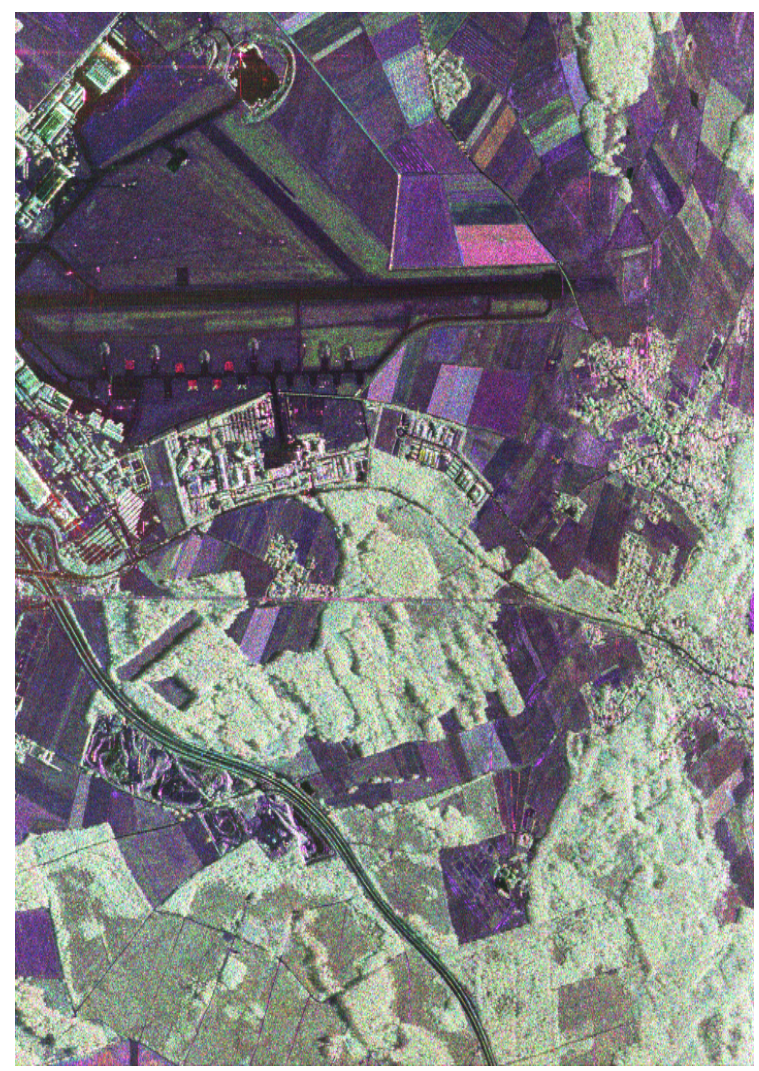

d)

Figure 1: Example of data sets used in the contest: a) high-resolution optical image (part of testsite Trudering), b) highresolution X-band SAR image (part of testsite Trudering), c) airborne optical image (testsite Oberpfaffenhofen), d) L-band polarimetric SAR image (testsite Oberpfaffenhofen). 


\section{EXAMPLES}

Figure 1 shows examples of data samples, which are going to be used in the contest. They give an impression of the mapping potential of optical and SAR data, both in a competitive as well as a complementary sense. Fig. 1 a) shows high-resolution optical data, acquired by an airborne sensor at a resolution of $0.4 \mathrm{~m}$. For comparison, in Fig. $1 \mathrm{~b}$ ), SAR intensity data of the testsite of Trudering/Germany, acquired by the commercial airborne X-band sensor AeS1 (2), is shown. This sensor reaches a resolution of about $40 \mathrm{~cm}$ and is, therefore, very well suited for mapping purposes. It demonstrates very well the high image quality that can be obtained with modern SAR sensors. Due to the high resolution, speckle effects are relatively small in this data, nevertheless it is clearly visible when looking at distributed targets like the agicultural fields. It can be expected, that the speckle problem in SAR imagery becomes much less important when reducing the resolution by multilooking techniques. With very high resolution systems, strong multilooking can be applied while still preserving an acceptable resolution.

In Fig. 1d) a polarimetric SAR image of the testsite of Oberpfaffenhofen/Germany is shown, acquired by the ESAR sensor of DLR (3). Here, the resolution is much lower (approx. $2 \times 2 \mathrm{~m}$ ), but the polarimetric system allows the generation of color images. Color coding often leads to a better mapping results, as the additional information helps in interpreting areas which are ambiguous in simple amplitude images. Finally, in Fig. 1 c) a panchromatic optical image of the testsite of Oberpfaffenhofen can be found. Clearly visible are the differences to the SAR image. Many areas appear more clear than in the SAR image, but other, like for example forested areas, are less differentiated in the optical image. Additionally, atmospheric artifacts become a problem, too.

Fig. 2 shows details of the polarimetric and the panchromatic image of Oberpfaffenhofen. The speckle problem in the SAR image becomes clearly visible at this magnification, but on the other hand on the man-made structures it can be observed that the true resolution of the SAR system is almost the same than the one of the optical image. One question to be answered in the contest is whether the bad visual impression of SAR data is only a subjective effect, or if the information content at the same resolution is really lower.

In Fig. 3, two other SAR data sets are shown, acquired by the danish EMISAR sensor in C-band. Both data set are fully polarimetric and represent two very special areas: An rural area around Fjordhundra in Sweden and the urban area of the city center of Copenhagen. This SAR data has a medium resolution of 1.5 meters, which should be enough for rural structures but which might be to bad to resolve adequately the finer urban stuctures, especially when appying multilooking for reducing the speckle effect. It should be shown by this contest which are appropriate resolutions for SAR and optical sensors, depending on the applications and scene type under investigation.
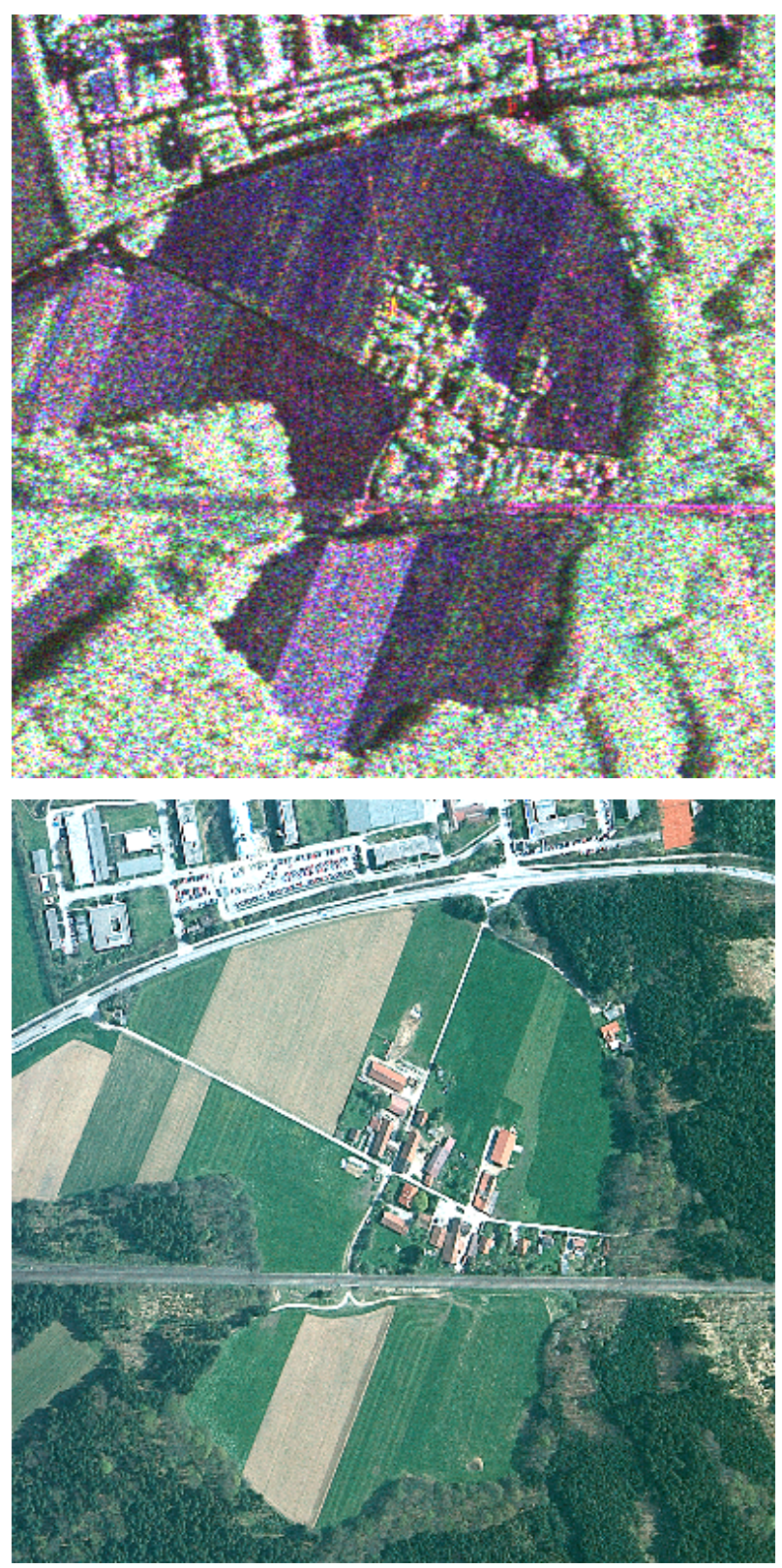

Figure 2: Speckled polarimetric SAR image (top) and smooth panchromatic optical image (bottom), testsite Oberpfaffenhofen/Germany

At the moment another testsite in Finland is under investigation, which might replace the Fjordhundra testsite. The Finland testsite has very well documented ground truth. Additionally, SAR and optical data of this site are easily accessible.

\section{PHASES OF THE CONTEST}

The test will be conducted in three phases. In phase 1, the participants will derive a visual interpretation of the data. The results will show the information content of the data independent of the performance of specific automatic object extraction methods and software. The data will be prepared at different resolution levels and distibuted to the participants. It is planned not to distribute SAR and op- 

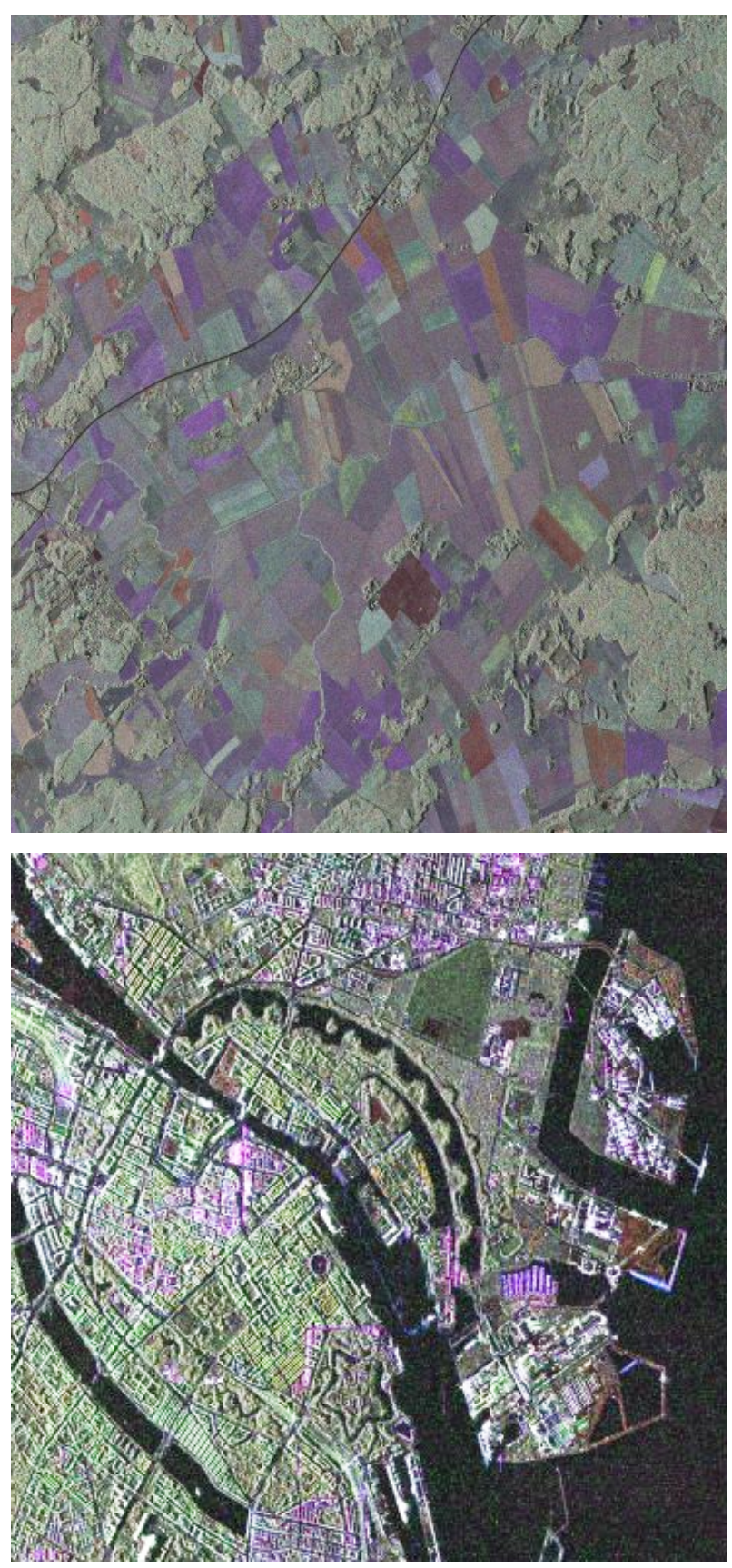

Figure 3: Polarimetric SAR images of testsite Fjordhundra/Sweden (top) and city center of Copenhagen (bottom).

tical data to the same parties in order to avoid undesired 'crosstalk' between the two data sets of a scene. A list of features to be extracted will be delivered together with the data sets. In this phase, mapping authorities, commonly active in state- or country-wide mapping projects, can give valuable contributions. Phase 1 will be started in autumn 2002.

In phase 2, automatic object extraction procedures will be applied to each sensor's data separately. Regarding data fusion, the results will be reference data for the performance of object extraction without use of multi-sensor data. They will, at the same time, represent the outcome of a competition of sensors. Probably, the question can be answered whether SAR is already an alternative means of data acquisition in mapping projects, compared with optical sensors, or whether it even outperforms optical sensors. In this phase, the alternative use of only partial data of one or both of the sensors may be requested from the participants. Object extraction results will be analyzed by comparison with the digital reference maps. In phase 2, any party (private company, governmental authority, university or research institute) can compete successfully by presentation of results, reflecting its specific expertise, e.g. in automatic extraction of a specific class of objects, or in compilation of maps fulfilling specific official requirements. Phase 2 will be started in spring 2003.

In phase 3, mapping will be conducted based on data of both sensors, i.e. by multi-sensor data fusion. This phase will give any participant, interested in research on data fusion, a chance to prove how valuable multi-sensor data fusion is, by demonstrating the degree of improvement in accuracy, correctness, completeness, robustness, or scope of object extraction. Phase 3 will be started in fall 2003.

\section{ACKNOWLEDGMENTS}

The authors have to thank several authorities for providing data for the contest, namely the the IHR/DLR (ESAR data), Aerosensing GmbH (AeS-1 data) as well as the DDRE (EmiSAR data).

\section{REFERENCES}

(1) O. Hellwich, C. Heipke, and B. Wessel: "Sensor and Data Fusion Con-test: Information for Mapping from Airborne SAR and Optical Imagery", Proceedings of IGARSS'2001, Sydney, pp. 2793-2795, 2001.

(2) F. Holecz, J. Moreira, P. Pasquali, S. Voigt, E. Meier and D. N uesch: "Height Model Generation, Automatic Geocoding and Mosaicing Using Airborne AeS-1 InSAR Data", Proceedings of IGARSS 97, pp. 1929-1931, Singapore, 1997.

(3) R. Horn: "DLR Airborne SAR Project: Objectives and Status", Proceedings of the First International Airborne Remote Sensing Conference, Strasbourg, France, 1994. 